\title{
Glottopol
}

Revue de sociolinguistique en ligne

$34 \mid 2020$

Les « langues de France ", 20 ans après

\section{De « langue régionale » à « langue de France » ou les ombres du territoire}

\section{Alain Viaut}

\section{(2) OpenEdition}

\section{Journals}

Édition électronique

URL : https://journals.openedition.org/glottopol/363

DOI : 10.4000/glottopol.363

ISSN : 1769-7425

Éditeur

Presses universitaires de Rouen et du Havre

Référence électronique

Alain Viaut, «De « langue régionale » à «langue de France » ou les ombres du territoire », Glottopol [En ligne], 34 | 2020, mis en ligne le 01 juillet 2020, consulté le 02 octobre 2021. URL : http:// journals.openedition.org/glottopol/363; DOI : https://doi.org/10.4000/glottopol.363

Glottopol 


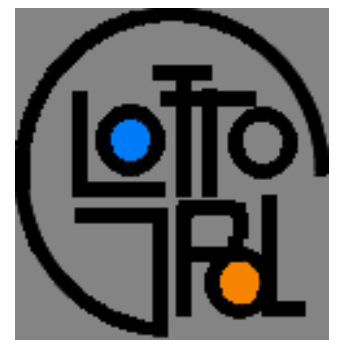

\section{GLOTTOPOL}

Revue de sociolinguistique en ligne $\mathrm{n}^{\circ} 34$ - juillet 2020

Les "langues de France " : 20 ans après

\section{SOMMAIRE}

Hommages à Jean Le Dî

Christian Lagarde : "Langues de France ». Au-delà du symbolique?

Entretien avec Bernard Cerquiglini, par Christian Lagarde.

Entretien avec Paul de Sinety, Délégué général à la langue française et aux langues de France, par Christian Lagarde.

Georg Kremnitz : La problématique initiale de la liste Cerquiglini et ses effets ultérieurs.

Alain Viaut : De "langue régionale » à "langue de France » ou les ombres du territoire.

Wanda Mastor: Le statut constitutionnel des langues régionales en droit comparé. De la reconnaissance à l'indifférence.

Philippe Martel, Marie-Jeanne Verny: Les langues régionales au Parlement, ou l'éternel retour.

Romain Colonna : Les «langues de France »: des langues non-étatiques au pays de l'Étatnation.

Hervé le Bihan : La langue bretonne : une visibilité toute en retenue.

Véronique Bertile : Les langues d'outre-mer : des langues de France? Approche juridique.

Jacques Vernaudon: Les langues polynésiennes et kanak, des "langues de France» en contexte de décolonisation.

Luc Biichlé : Qu'advient-il de l'arabe de France? Mise en perspective sociolinguistique...

Pascal Ottavi : L'épervier, la cage et le passereau.

Marielle Rispail : Le francique lorrain, langue de France? Réflexions et témoignages.

\section{Compte rendu de lecture}

Par Salih Akin : Jean Le Dû \& Yves Le Berre, Métamorphoses. Trente ans de sociolinguistique à Brest (1984-2014), Brest, Centre de Recherche Bretonne, 2019, 302 p.

http://glottopol.univ-rouen.fr 


\title{
DE « LANGUE RÉGIONALE » À « LANGUE DE FRANCE » OU LES OMBRES DU TERRITOIRE
}

\author{
Alain Viaut \\ UMR 5478 Iker (CNRS - Université Bordeaux Montaigne - UPPA)
}

Les désignants «langue régionale» et «langue de France» réfèrent d'emblée à l'implantation proprement territoriale de langues. Il s'agit de langues qui s'inscrivent historiquement, depuis une durée plus ou moins significative, voire courte comme, par exemple, pour le hmong en Guyane, initialement langue d'immigration diasporique récente. L'adjectif « régionale » qualifie telle langue selon un mode géographique en se référant à une portion infraterritoriale d'un État, ici la France. «De France » opère dans le même sens puisque la France est un territoire avec ses frontières mais en apportant une nuance politique, celle de l'appartenance à la fois fractionnelle - une des langues de France - et une acception plus large que celle qui découle de l'inscription spatiale. De fait, à ce sème-là s'ajoute celui, plus abstrait, de la partie constitutive, qui n'est pas forcément inscrite dans, justement, une "région ». Or l'identification de ces parties constitutives renverra alors à ce qui est entendu par « France », avec ses tenants d'histoire et de culture politique, par les concepteurs de la notion de «langue de France », toutes choses dont nous allons tenter une approche dans ce qui suit.

La notion de langue régionale est apparue pour la première fois en France, dans un cadre juridique, en 1966, dans la circulaire $n^{\circ} 66-361$ du 24/10/1966 (BO n 41 du 03/11/1966) portant création des Commissions académiques d'études régionales. Celles-ci furent mises en place pour étudier « les divers problèmes théoriques et pratiques que pose l'enseignement des langues régionales ». Le terme de " langue régionale » ainsi formulé sera présent par la suite, jusqu'à aujourd'hui, dans les divers textes règlementant la place des langues concernées dans l'enseignement scolaire, de la circulaire à la loi (loi Haby 1975, loi Jospin 1989, loi Toubon 1994, codifiées ensuite dans le Code de l'Éducation, de 2000). Avant que cette formulation ne devienne habituelle, on pourra cependant noter que, dans la circulaire $n^{\circ}$ IV-69-90 du 17/02/1969 sur l'Enseignement des langues et cultures régionales dans les classes des premier et second degrés, qui initia véritablement la première mise en application réelle de la loi Deixonne $\mathrm{n}^{\circ}$ 51-46 du 11/01/1951 relative à l'enseignement des langues et dialectes locaux ${ }^{1}$, « langues régionales » figurait à part dans une partie spécifique intitulée «Enseignement des langues régionales ». La formulation « langue régionale » fut également employée, au singulier ou au pluriel, dans la deuxième partie de cette circulaire intitulée «Enseignement des

\footnotetext{
1 Abrogée depuis et dont le contenu fut intégré au nouveau Code de l'Éducation (https://www.cjoint.com/doc/20 01/JAhmMVvMiTh_codeeducation.pdf, consulté le 29/02/2020).
} 
civilisations régionales », en relation explicite ou implicite avec les différentes disciplines avec lesquelles on l'associait (histoire, français, enseignement artistique) pour l'enseignement du Premier degré, ainsi qu'avec les programmes des deux cycles des programmes du Second degré.

\section{De « langue régionale » à « langue et culture régionales »}

Par la suite, la circulaire $\mathrm{n}^{\circ}$ 71-279 du 07/09/1971 continuera de mettre l'accent sur l'enseignement des « langues régionales » en tant que telles tout en les incluant également, par son intitulé, dans l'«enseignement des langues et cultures régionales dans les classes des premier et second degrés ». L'association des deux objets d'enseignement colloquant en un même syntagme «langues régionales » et "cultures régionales", deviendra habituelle, en revanche, par la suite, au point d'apparaitre comme la notion désormais aussi courante que celle de «langue régionale » dans la pratique du Ministère de l'Éducation nationale. Cela ne s'accompagnera pas pour autant de l'autonomisation des «cultures régionales » comme objet d'un enseignement autonome. Les cultures régionales demeureront ainsi presque exclusivement liées aux langues qualifiées de manière identique en une expression qui se figera pour former une notion propre au Ministère de l'Éducation nationale en France, quasi prototypique dans ce champ d'application, autant que celle de « langue régionale ». Pour exemple, dans le Décret du Premier ministre $\mathrm{n}^{\circ} 2001-733$ du 31/05/2001, portant Création d'un Conseil académique des langues régionales, « langue et culture régionales » apparait 10 fois, et « langue régionale » 6 fois si l'on omet les occurrences dans lesquelles le terme est contenu dans le rappel à plusieurs endroits de l'intitulé dudit Conseil académique. L'ancienne formulation « langues et dialectes locaux » de la Loi Deixonne de 1951 (cf. supra), persistera cependant dans les textes réglementaires qui continueront à en appliquer les dispositions jusqu'au début des années $1990^{2}$. Cette loi sera abrogée en 2000 par l'Ordonnance $n^{\circ} 2000-549$ du 15/06/2000 relative à la partie législative $d u$ Code de l'Éducation ${ }^{3}$, et ses dispositions ainsi que celles qui concernaient les « langues et dialectes locaux » ou «langues régionales » contenues dans des textes législatifs antérieurs seront reportées en 2000 dans le nouveau Code de l'Éducation (2020 [2000]).

\section{Langue régionale, propriétés définitoires}

Quoi qu'il en soit, au-delà du champ politique et administratif, "langue régionale » se répandra dès lors comme un désignant commun en France à partir de ces années 1970. Il n'en demeure pas moins que la notion est devenue celle qui fait référence pour l'administration du ministère de l'Éducation nationale. Elle y désigne le type de langues qui y fait l'objet depuis cette époque d'un appareil de mesures juridiques. Ainsi que nous le verrons plus avant, on la trouve également utilisée dans un autre cadre institutionnel supérieur depuis 2001. Il s'agit de celui du ministère de la Culture où elle apparait depuis lors pour dénommer une sous-catégorie des « langues de France » (cf. infra). Rappelons-le néanmoins, la notion catégorisante « langue régionale » ne s'est appliquée au domaine, tout d'abord de l'enseignement puis, secondairement, de la culture, en s'appuyant sur les critères d'historicité et d'implantation territoriale, qu'à partir de 1966 en France à cet échelon officiel, et en application de la loi

\footnotetext{
${ }^{2}$ Ainsi du Décret 92-1162 du 20/10/1992, Relatif à l'enseignement des langues et dialectes locaux (JO 23/10/1992, p. 14767) : «Les articles 2 à 9 de la loi du 11 janvier 1951 modifiée relatifs à l'enseignement des langues et dialectes locaux sont applicables dans la zone "influence des langues mélanésiennes pour ce qui concerne l'ajië, le drehu, le nengone et le paicî.” " (Eysseric, 2005).

${ }^{3}$ https://www.legifrance.gouv.fr/affichTexte.do?cidTexte=JORFTEXT000000583540 (consulté le 29/02/2020).
} 
Deixonne de 1951. Or, celle-ci, dès son article 1, précisait qu'elle avait pour objectif de " favoriser l'étude des langues et dialectes locaux dans les régions où ils sont en usage ». Et il était précisé, dans son article 5 concernant les écoles normales et les enseignants intéressés, que ces derniers étaient destinés «à enseigner dans une région où une langue locale a affirmé sa vitalité ». Le territoire d'implantation et l'affirmation de la « vitalité » de la langue impliquée étaient ainsi mis en évidence dans ce texte premier.

Or, si la vitalité linguistique n'apparait pas ensuite à travers cette formulation, on peut la supposer sous-jacente lorsque les langues régionales deviennent éligibles à leur enseignement dans la mesure où elles font l'objet de demandes manifestes de la part des intéressés (parents d'élèves, élèves eux-mêmes). Le territoire d'implantation, de son côté, apparaitra régulièrement, à travers le syntagme « les académies où les langues régionales sont en usage » ${ }^{4}$, avec le désignant «zone d'influence " ${ }^{5}$, censé autoriser une signification plus large que l'usage qui peut être réel sous les formes orale, écrite ou les deux dans «les aires où la langue correspondante est traditionnellement pratiquée $»^{6}$. De fait, alors que ces langues ont vu leur usage continuer à reculer après les années 1970, le Code de l'Éducation de 2000 s'appuie encore sur la référence à l'aire d'usage (article L312-10), de même que plus tard, par exemple, la loi $\mathrm{n}^{\circ}$ 2000-1207 du 13/12/2000 d'orientation pour l'outre-mer (in article $34:$ : les langues régionales en usage dans les départements d'outre-mer ») ou la décision du Conseil d'État $n^{\circ} 316946$ du 09/12/2009 qui contribue à caractériser la nature d'une langue régionale en Droit français à partir de son implantation spatiale et de son caractère minoritaire. Cette décision indique qu'une langue telle que l'anglais - dont la situation minoritaire ne pourrait être valablement invoquée ici - ne saurait être « considérée comme une langue régionale en usage en France ${ }^{7}$. On pourra alors inférer de cette allégation 1'hypothèse selon laquelle l'implantation territoriale comporte en l'espèce, parmi ses sèmes sous-jacents, ceux d'historicité et de patrimoine, ce dernier étant compris ici comme étant constitutif de celui global de la France.

Les sèmes d'historicité et de patrimoine se retrouvent explicitement dans d'autres textes à contenu juridique et intègrent de fait la signification spontanément comprise de "langue régionale ». Déjà, la circulaire n ${ }^{\circ} \mathrm{IV}-69-90$ du 17/02/1969 sur l'Enseignement des langues et cultures régionales dans les classes des premier et second degrés en fait état dans son préliminaire : «Elles [les langues et cultures régionales] font partie, en effet, ainsi que les civilisations dont elles sont l'expression, du patrimoine national et fournissent des éléments pédagogiques du plus grand intérêt ». Cela continuera et, une trentaine d'années après, le lien explicite entre langue régionale et patrimoine ne sera pas démenti. La circulaire $\mathrm{n}^{\circ}$ 2001-166 du 05/09/2001 Sur le développement de l'enseignement des langues et cultures régionales à l'école, au collège et au lycée assigne ainsi, comme premier objectif de ses orientations générales, « préservation et transmission d'un élément de la richesse du patrimoine national ». Le deuxième objectif étant la «contribution à la reconnaissance de la diversité culturelle au sein de la communauté nationale», cela rappelle la notion voisine de celle de «langue

\footnotetext{
${ }^{4}$ Ainsi, pour les années 1960, dans la circulaire ${ }^{\circ}$ 66-361 du 13/10/1966 (Création des Commissions académiques d'études régionales), circulaire n ${ }^{\circ}$ IV-69-90 du 17/02/1969 (Enseignement des langues et cultures régionales dans les classes du premier et second degrés).

${ }^{5}$ In décret $\mathrm{n}^{\circ}$ 74-33 du 16/01/1974 étendant le champ d'application de la loi Deixonne de 1951 à la langue corse.

${ }^{6}$ In circulaire no 76-123 du 29/03/1976 sur la Prise en compte, dans l'enseignement, des patrimoines culturels et linguistiques français (partie "Langue et dialectes locaux").

7 Considérant que, contrairement à ce que la société soutient par ailleurs, la langue anglaise ne saurait être considérée comme une langue régionale en usage en France, alors même qu'elle est parlée par certains habitants des Antilles françaises ; Considérant qu'il résulte de tout ce qui précède que la société MARGO FILMS n'est pas fondée à demander l'annulation de la décision attaquée (considérants $\mathrm{n}^{\circ} 5$ et 6 , https://www.legifrance.gouv.fr/affichJuriAdmin.do?idTexte=CETATEXT000021468369, $\quad$ consulté le 29/02/2020).
} 
régionale », qui s'est développée en parallèle à partir des années 1970 également, quoiqu'elle ne fût pas encore exprimée de façon claire ni dans le texte de la loi Deixonne de 1951, ni dans celui de la circulaire de 1966 instituant les Commissions académiques d'études régionales. Il s'agit de celle de « langue et culture régionale » dont la formulation s'est figée à partir de là. On peut cependant considérer qu'elle était en germe dans les articles 4 , sur les ouvrages portant sur les « richesses culturelles et le folklore des régions dans les bibliothèques scolaires » ${ }^{8}$, et 5 , sur les cours et les stages « le folklore, la littérature et les arts populaires locaux dans les écoles normales ${ }^{9}$ de la loi Deixonne.

Déjà, les circulaires n IV-69-90 du 17/02/1969 (cf. supra) et 71-279 du 07/09/1971 s'y réfèrent dans leur intitulé lui-même : "Enseignement des langues et cultures régionales dans les classes des premier et second degrés ». Le lien entre la langue et la culture est explicitement détaillé dans le premier de ces deux textes et concerne la relation qui est mise à contribution entre, précisément, " les académies où les langues régionales sont en usage » et "l'étude de la civilisation qui s'y rattache ». En réalité, la circulaire de 1969 recommandait non un enseignement en une seule discipline de la langue et de la culture régionales mais de la langue régionale en tant que telle et, dans l'enseignement du premier degré, des «civilisations régionales » insérées dans les cours d'histoire, de « géographie régionale et locale ${ }^{10}$, de français et d'enseignement artistique. L'incitation à l'insertion des « civilisations régionales » ou, par la suite, selon la formule standardisée, des « langues et cultures régionales » dans ces disciplines sera répétée dans la circulaire $n^{\circ} 71-279$ du 07/09/1971. En fait, cela continuera et se retrouvera jusqu'à aujourd'hui selon cette modalité-là. La circulaire $n^{\circ}$ 2001-166 du 05/09/2001 en rappela ainsi le principe dans ses orientations générales («acquisitions culturelles liées au patrimoine dans lequel s'inscrit la langue (histoire, géographie, littérature, arts, etc. ») comme, précisément, pour les écoles élémentaires en association, en outre, avec «l'enseignement de toutes autres langues vivantes ». Cela est enfin présent dans la circulaire plus récente encore $\mathrm{n}^{\circ} 2017-072 \mathrm{du}$ 12/04/2017 relative à l'enseignement des langues et cultures régionales ${ }^{11}$, et est étendu aux enseignements concernés du second degré ainsi que pour « favoriser l'acquisition du socle commun de connaissances, de compétences et de culture et des programmes scolaires $»$.

Sous ces rapports, cette configuration générale qui a progressivement été installée en suivant des trajectoires politiques voulues ou consenties et un inévitable empirisme, dont les éléments sont repris dans le Code de l'Éducation de 2000, aboutit à établir l'emploi de l'adjectif régional dans un tel contexte pour une double référence. Celle de « langue régionale » désigne en France une catégorie de langue minoritaire concernée par une place spécifique dans l'enseignement public, différente de celle du français et de celle des langues étrangères, même si elle a parfois été associée à cette dernière au titre de langue II ou III. Elle désigne également l'espace de l'enseignement bilingue facultatif français / langue régionale ${ }^{12}$. L'autre référence, celle de « langue et culture régionales », s'applique à l'espace dans lequel l'enseignement public de la

\footnotetext{
${ }^{8}$ «Les maitres seront autorisés à choisir, sur une liste dressée chaque année par le recteur de leur académie, les ouvrages qui, placés dans les bibliothèques scolaires, permettront de faire connaitre aux élèves les richesses culturelles et le folklore de leur région. »

${ }^{9}$ «Dans les écoles normales, des cours et stages facultatifs seront organisés, dans toute la mesure du possible, pendant la durée de la formation professionnelle, à l'usage des élèves-maitres et des élèves-maitresses qui se destinent à enseigner dans une région où une langue locale a affirmé sa vitalité. Les cours et stages porteront, non seulement sur la langue elle-même, mais sur le folklore, la littérature et les arts populaires locaux. »

10 «Les programmes et instructions en vigueur prescrivent cet enseignement», est-il précisé sous le nom de la discipline.

${ }^{11} \mathrm{http}$ ://circulaires.legifrance.gouv.fr/pdf/2017/04/cir_42043.pdf (consulté le 29/02/2020).

${ }^{12} \mathrm{Cf}$. article L312-10 $2^{\circ}$ ) du Code de l'Éducation.
} 
langue régionale, en lien avec son contexte historico-culturel, prend place en tant que discipline facultative ${ }^{13}$.

De ce premier regard jeté sur les textes fondateurs de la notion de «langue régionale » en France, on retiendra les propriétés définitoires suivantes qui sont autant de traits. Il s'agit, dans ce contexte français, d'une langue minoritaire, sur le plan démolinguistique, à la fois à l'échelle du pays et aussi à l'intérieur de son territoire d'implantation, qui, comme ailleurs, s'inscrit dans une portion particulière du territoire du pays. Le mot région et son dérivé adjectival classifiant, reflètent cette réalité. Le sème de territoire subordonné et politiquement régi par une entité compétente à un échelon plus vaste (regere $>$ regio $>$ regionalis) est constitutif du sens de " régional ». Dans le contexte français, cette portion, également minoritaire, territorialement, est celle d'une langue qui l'est d'abord par son statut juridique, social et démolinguistique. En effet, le paramètre spatial pris seul ne saurait être avancé que de manière nuancée. Si l'on prend l'exemple de l'occitan, l'aire marquée par cette langue, la plus importante de celles qui sont concernées par d'autres langues régionales en France métropolitaine, représente une proportion significative de ce pays, soit un gros tiers. En outre, la langue régionale se caractérise aussi par l'historicité de son implantation dans un territoire. On la fait remonter à une période indéterminée avec précision mais très ancienne dans le cas du basque, à la période préromaine et du Haut Moyen Âge dans celui du breton (gaulois + britonnique) et, dans leurs fondements, au Haut Moyen Âge aussi et à la période précédant l'an 1000 pour celle des langues germaniques et romanes minoritaires.

Cette ancienneté est même précisée par la Délégation générale à la langue française et aux langues de France (DGLFLF désormais) du ministère de la Culture. Les langues régionales, pour être reconnues comme telles, doivent être «parlées sur une partie du territoire national depuis plus longtemps que le français langue commune $»^{14}$. De ces points de vue, la définition donnée par le Dictionary of Sociolinguistics (Swann et al., 2004) pour regional language, qui met l'accent sur l'aspect géographique, l'historicité du territoire d'implantation et sur la subordination à une autre langue ${ }^{15}$, rappelle, au-delà de la France, la diffusion de ces propriétés définitoires. Or, en partant du cas français, ces propriétés estimées être pertinentes ici rejoignent celles que nous avions définies comme possédant une qualification identique à un niveau plus large, au moins européen au sens large (Viaut \& Pascaud, 2017 : § 20-23).

\section{La langue régionale et les nuances du patrimoine}

Toutefois, dans ce que venons d'observer, l'historicité se trouve corrélée à l'idée de patrimoine, propriété que nous déduisons comme étant également pertinente mais secondaire de la notion associée de «langue et culture régionales » (Viaut \& Pascaud, 2017 : § 45-46). Le lien entre « langue régionale » avec même, dès le début, cette formulation, et «culture » nous a paru être substantiellement sous-jacent. L'enseignement de la langue régionale, dans le texte de la loi Deixonne impliquait cela, la circulaire n IV-69-90 du 17/02/1969 sur 1'Enseignement des langues et cultures régionales dans les classes des premier et second degrés de même, à côté du réglage "technique » qui avait consisté à distinguer l'enseignement de la langue régionale - et, plus tard aussi, en langue régionale - de celui des «langues et cultures

\footnotetext{
${ }^{13}$ Cf. article L312-10 $1^{\circ}$ ) du Code de l'Éducation.

14 https://www.culture.gouv.fr/Sites-thematiques/Langue-francaise-et-langues-de-France/Politiques-de-lalangue/Langues-de-France/Langues-regionales, consulté le 29/02/2020).

15 « Regional language: a language which is spoken in a specific geographical region of a country but not across the entire political territory. Regional languages are rarely recognized in the national constitution of a country but are sometimes included in education policies. (...)» (Swann et al., $2004:$ 260-261).
} 
régionales ». Pour autant, deux conceptions du lien entre langue régionale et patrimoine se firent jour en France entre les années 1970 et 2000, entre deux visions, statique et dynamique, de ce que peut représenter le patrimoine linguistique, également conçu pour s'appliquer à une langue répandue comme le français, ce dernier étant régulièrement proclamé comme une des expressions les plus hautes du patrimoine de la France. D'un côté, une conception, qui se dégage de la circulaire $\mathrm{n}^{\circ} 76-123$ du 29/03/1976 sur la Prise en compte dans l'enseignement, des patrimoines culturels et linguistiques français, reconnait le patrimoine culturel sous ses différents aspects « notamment linguistiques », conçu comme étant représentatif de la " somme des rapports particuliers entre le sol et les hommes qui s'y sont succédés ", sans qu'il soit fait état de la place particulière des patrimoines liés en propre à chacune des langues régionales mais, surtout, pour que :

L'étude des différents patrimoines culturels et linguistiques et de leurs apports spécifiques à ce que fut aux différentes époques de notre histoire, la culture française, doit montrer la variété et la richesse des éléments qui se sont amalgamés pour constituer notre personnalité nationale. (Partie introductive, dernier paragraphe)

D'un autre côté, une vision plus ouverte a pu être assignée par la suite - clairement à partir des années 1990 - au binôme langue et culture, explicitement ou implicitement lié à l'idée légitimante de patrimoine, avec l'introduction du mot «promotion » dont la signification est empreinte de dynamisme. Cela s'inscrit dans une tendance plus large, dont des échos sont perceptibles dans des textes européens. Un des paragraphes du préambule de la Charte européennes des langues régionales ou minoritaires $d u$ Conseil de l'Europe (désormais CELROM) mise à la signature en 1992, énonce que « la protection et la promotion des langues régionales ou minoritaires dans les différents pays et régions d'Europe » contribue à la démocratie et à la diversité culturelle. Dans cet esprit également, la Résolution sur les minorités linguistiques et culturelles dans la Communauté européenne, votée le 09/02/1994 par le Parlement européen affirme, dans son paragraphe B, que la promotion d'une culture linguistique européenne doit s'accompagner de «l'élimination des entraves linguistiques, la promotion des langues de moindre diffusion et la sauvegarde des langues minoritaires, (...) ».

Cette tendance est confirmée aussi, finalement, par le Code de l'Éducation (2020 [2000]) en France. Nous sommes ainsi enclins à lire dans ce sens l'article L123-6 qui assigne pour mission au service public de l'enseignement supérieur la mission de "veiller à la promotion et à l'enrichissement de la langue française et des langues et cultures régionales ». De même, dans l'article D312-36 : le Conseil académique des langues régionales, dans sa contribution à « la définition d'une politique d'édition, de production et de diffusion du matériel pédagogique pour l'enseignement de la langue régionale », « il est tenu informé des conventions passées (...) avec les associations concourant à la promotion des langues et cultures régionales ».

Nous en venons à inférer que du lien intrinsèque entre langue régionale, culture et patrimoine, ce dernier peut aussi être considéré comme une propriété définitoire, même si seconde. L'article 75-1 de la Constitution, issu de la loi constitutionnelle du 23/07/2008 consacre à la fin cela en stipulant que : «Les langues régionales appartiennent au patrimoine de la France ». Au-delà, on peut aussi estimer que ce lien est au moins implicite hors de France, et, sans entrer ici dans plus de détails, il est présent dans de nombreux autres pays d'Europe au sens large, de l'Espagne à la Russie (Viaut \& Pascaud, 2017 : 10). 


\section{Langue régionale, propriétés afférentes}

Pour le reste, le contexte proprement français est naturellement à la source de propriétés afférentes pour la notion de langue régionale qui sont liées à son développement notoire en France. Quatre propriétés afférentes sont proposées comme ce qui suit.

La langue régionale se définit selon le ministère de la Culture, depuis 2001, par sa distance chronologique et linguistique avec le français standard. Ont ainsi été définis comme langues régionales les dialectes primaires de la langue d'oïl (au même titre que le basque ou l'occitan) parce qu'ils étaient parlés depuis plus longtemps que le français standard, comme les dialectes, également constitutifs, de l'occitan, non coiffé, lui, par une variété commune (cf. supra, sur l'historicité des langues régionales) ainsi que cela est expliqué dans le texte de présentation de la liste des langues régionales par la DGLFLF du ministère de la Culture ${ }^{16}$. Distance linguistique avec le français standard, l'argument est avancé par Bernard Cerquiglini en 1999 dans son Rapport au ministre de l'Éducation, de la Recherche et de la Technologie sur les «Langues de la France», pour joindre les langues d'oïl (langues Ausbau sur la base des dialectes primaires du français) à la liste des langues régionales et minoritaires au moment où la France s'apprêtait à ratifier la CELROM :

Il en découle également que l'écart n'a cessé de se creuser entre le français et les variétés de la langue d'oill, que l'on ne saurait considérer aujourd'hui comme des «dialectes du français»; franc-comtois, wallon, picard, normand, gallo, poitevin-saintongeais, bourguignon-morvandiau, lorrain doivent être retenus parmi les langues régionales de la France; on les qualifiera dès lors de «langues d'oïl », en les rangeant dans la liste (Cerquiglini, 1999 : « Les dialectes de la langue officielle »).

La langue régionale n'est éligible, dans le domaine public et la sphère administrative, que dans l'enseignement, de la maternelle à l'Université. Or cela ne constitue pas un droit mais relève de la volonté des autorités publiques (Bertile, $2010: 78$ ). Il n'y a pas, à ce jour, de droit à proprement parler en France à l'enseignement d'une langue régionale, celui-ci continuant à découler de son caractère facultatif en droit. Les droits linguistiques qui en résultent pour les locuteurs de langue régionale ou/et ceux qui affirment leurs liens avec elles sont d'ordre culturel, et « en tant que tels (...) se présentent comme des droits à la différence et contribuent à l'épanouissement de la personne humaine »(Bertile, $2010: 81)^{17}$. Les autres possibilités dans le domaine public (administration locale, régionale, signalisation publique) sont par principe fermées aux langues régionales, sauf exception tolérée comme, par exemple, pour les panneaux d'entrée et de sortie des chefs-lieux de commune dont les noms, référencés par l'INSEE et le Code officiel géographique, ne sont révisables qu'après décision du Conseil d'État mais qui, depuis ces dernières décennies, peuvent porter mention de leur nom sous une forme linguistique propre en dessous du nom officiel généralement francisé. Il peut en aller de même des noms infracommunaux (rues, places, etc.) dans le cadre de la liberté communale. Les autres collectivités territoriales, régions et départements concernés, ont parfois mis en pratique une signalétique interne bilingue dans les bâtiments des Conseils régionaux ou départementaux. Du reste, cela peut aussi être le cas de certaines communes, dans les mairies et bâtiments municipaux, comme en Pays basque. En revanche, paradoxalement, la toponymie des cadastres

16 https://www.culture.gouv.fr/Sites-thematiques/Langue-francaise-et-langues-de-France/Politiques-de-lalangue/Langues-de-France/Langues-regionales (consulté le 29/02/2020).

17 Véronique Bertile définit en outre ces droits linguistiques en France comme des droits «prestationnels» (l'individu seul ne peut y satisfaire, et l'État est censé l'aider en cela), des « droits-devoirs » (ils doivent se concilier avec le principe constitutionnel instaurant une seule langue officielle, et des droits territoriaux (circonscrits à un espace géographique) (Bertile, $2010: 81-82$ ). 
communaux peut être révisée et se présenter officiellement sous sa seule forme linguistique propre, après accord entre les communes demandeuses, assistées d'organismes scientifiques référencés, et l'Institut géographique national (IGN) (Lejeune, 2008), comme cela s'est produit en Roussillon pour le catalan au début des années 2000 (Becat, 2008).

Associées à cette première propriété afférente française, les modalités de l'enseignement sont toujours optionnelles ou facultatives, quoiqu'avec des nuances dans l'application de la réglementation quant aux modalités concrètes de la proposition d'enseignement.

La langue régionale n'a pas, par définition, de statut d'officialité ou de coofficialité de jure ou de facto. Un premier pas dans ce sens qui consisterait à pouvoir accéder, même de façon réduite ou symbolique, à un usage public lié à des fonctionnalités politico-administratives ne serait-ce que locales, n'existe que de manière très réduite (cf. supra), à la différence, en particulier, de ce qu'il en est des possibilités proposées à cet égard par la CELROM.

L'emploi lui-même de la formulation «langue régionale» est significatif d'un choix restreint des dénominations catégorisantes acceptées ou acceptables. C'est probablement une des raisons de sa diffusion, plus par en haut que par en bas d'ailleurs. Des dénominations se focalisant sur le groupe locuteur telles que "minorité linguistique » ou «communauté linguistique », ou bien comportant un qualificatif renvoyant directement au type sociolinguistique de langue en relation avec un groupe telles que « langue nationale », « langue ethnique », voire même «langue minoritaire » (qui réfère à minorité et, par conséquent, à groupe), en usage au gré de chacun, n'est cependant guère possible en tant qu'usage juridique, comme le rappela le Conseil constitutionnel, se prononçant négativement à propos de la ratification par la France de la CELROM dans le sixième considérant de sa décision $\mathrm{n}^{\circ}$ 99-412 DC du 15/06/1999 : "Considérant que ces principes fondamentaux [ceux contenus dans l'article 1 de la Constitution ${ }^{18}$ ] s'opposent à ce que soient reconnus des droits collectifs à quelque groupe que ce soit, défini par une communauté d'origine, de culture, de langue ou de croyance $»^{19}$.

\section{Les « langues de France» ou « la langue est dans le cerveau de celui qui la parle »}

Quelque trente-cinq ans plus tard, la notion de « langue de France » apparaitra dans un autre cadre formel que celui de l'Éducation nationale, en 2001, avec le décret $n^{\circ}$ 2001-950 du 16/10/2001 instituant, au sein du ministère de la Culture, la Délégation générale à la langue française et aux langues de France (DGLFLF) «modifiant le décret n 89-403 du 02/06/1989 par lequel avait été créés le Conseil supérieur de la langue française et la Délégation générale à la langue française ». Auparavant, dans l'article 21 de la loi du 04/08/1994 relative à l'emploi de la langue française, il avait été question des "langues régionales de la France » : "Les dispositions de la présente loi s'appliquent sans préjudice de la législation et de la réglementation relatives aux langues régionales de France et ne s'opposent pas à leur usage $»^{20}$.

C'est en fait au moment où la France s'apprêtait à adhérer à la CELROM que la notion prit sa forme définitive. Ainsi, dans son rapport préparatoire à cette adhésion, Bernard Poignant parle des formules qu'il aurait souhaité voir figurer dans la Constitution française en faveur des langues régionales en les désignant comme des « langues régionales ou historiques de France » ou comme des «langues historiques du peuple de France » (Poignant 1998: 40). La même année, dans son rapport également remis au premier ministre avec la même finalité, le juriste

\footnotetext{
18 «La France est une République indivisible, laïque, démocratique et sociale. Elle assure l'égalité devant la loi de tous les citoyens sans distinction d'origine, de race ou de religion. Elle respecte toutes les croyances ».

${ }^{19} \mathrm{https}$ ://www.conseil-constitutionnel.fr/decision/1999/99412DC.htm (consulté le 29/02/2020).

${ }^{20} \mathrm{https}$ :/www.legifrance.gouv.fr/affichTexte.do?cidTexte=LEGITEXT000005616341 (consulté le 29/02/2020).
} 
constitutionnaliste Guy Carcassonne (1998) donne, comme alternative synonyme de langue régionale, "langue de la France », déjà dans son intitulé, et "langue régionale de France ». L'année suivante, le linguiste Bernard Cerquiglini (1999), dans un troisième rapport censé préparer aussi cette adhésion à la CELROM, reprend la désignation "langues de la France », qui sera consacrée, deux ans après par le ministère de la Culture comme nous l'avons vu, sous la forme plus concise et, par conséquent, figée et emblématique, «langues de France ».

Comme l'a montré Véronique Bertile (2010: 74), il apparait bien que le terme « langue de France » permettait de décrocher en quelque sorte cette catégorie sociolinguistique de son ancrage territorial néanmoins conçu comme évident jusque-là. Bernard Cerquiglini écrit dans son rapport que «le vrai territoire d'une langue est le cerveau de ceux qui la parlent». Le contexte est d'un côté celui du rejet politique des "communautés », issues de l'immigration comme liées à des langues régionales. La défiance à l'égard des territoires des langues régionales est en même temps argumentée avec un réalisme sociolinguistique :

à la réalité sociolinguistique, qui rappelle que la mobilité sociale contemporaine est telle que l'on parle les différentes langues "régionales » un peu partout. Le créole est une réalité linguistique bien vivante de la région parisienne. Signant puis ratifiant la Charte, la République française aurait donc intérêt, dans sa déclaration, à insister sur la vocation culturelle de la Charte, en minorant la tendance à la territorialisation. (Cerquiglini 1999, sous « territoire »)

Ce rapport au territoire est critiqué par Guy Carcassonne et Bernard Cerquiglini comme étant une caractéristique de la CELROM dont la France devait se défier au nom de son universalisme et d'un égalitarisme entre langues minoritaires, lesquelles devaient inclure des «langues dépourvues de territoire ». Le mot territoire est, en effet, bien présent (19 fois) dans le texte de la CELROM, et il s'agit autant de l'entité spatiale historico-culturelle que de gestion obligée, administrative, des langues concernées. Certaines langues d'immigration, en fait, celles qui ne bénéficient pas de protection dans leur pays d'origine en tant que langues officielles d'État et qui ont des liens historiques avec la France sont adjointes aux autres langues de France dans le rapport de Bernard Cerquiglini. La liste qui résultera de son approche ne figurera pas dans la déclaration adressée le 07/05/1999 par la France au Conseil de l'Europe avec sa signature de la CELROM, et le seul désignant « langue régionale ou minoritaire » propre à cette convention y est utilisé ${ }^{21}$.

Au résultat, cette notion de «langue de France », telle qu'elle est définie sur le site de la DGLFLF (Sibille, 2010, 2013), est plus large que celle de "langue régionale », telle qu'entendue par le ministère de l'Éducation nationale, puisqu'elle comprend les trois souscatégories suivantes : les « langues régionales » dont la liste est plus longue que celle dont traite de ministère de l'Éducation nationale ${ }^{22}$, les « langues des Outre-mer » et une série de « langues non-territoriales » qui reprend celle qu'avait retenue Bernard Cerquiglini dans son rapport ${ }^{23}$.

On aurait pu déduire de ce rapport hyperonymique que la notion de «langue de France » se serait imposée comme celle qui coifferait celles de «langue régionale » et d'autres plus spécifiques. Elle est utilisée dans le cadre valorisant de l'agrégation précisément « des langues

21

https://www.coe.int/fr/web/conventions/full-list//conventions/treaty/148/declarations?p_auth $=$ vUoelhkB\&_coeconventions WAR coeconventionsportlet enVig ueur $=$ false\&_coeconventions_WAR_coeconventionsportlet_searchBy=state\&_coeconventions_WAR_coeconve ntionsportlet codePays $=$ FRA ${ }^{\&}$ coeconventions WAR coeconventionsportlet codeNature $=10$ (consulté le 29/02/2020).

${ }^{22}$ En particulier parce que celle-ci inclut le franco-provençal et les langues d'oïl, non prises en compte, hormis le gallo, par l'Éducation nationale.

${ }^{23}$ L'arabe dialectal (dans ses diverses variétés), l'arménien occidental, le berbère, le judéo-espagnol, le romani et le yiddish. 
de France », de création récente, en 2017, et qui s'applique à des langues régionales (basque, breton, catalan, corse, créole, occitan-langue d'oc, tahitien), logiquement celles qui étaient déjà éligibles au CAPES de langues régionales et du corse depuis une vingtaine d'années. Or, la formule qui fut retenue par la loi constitutionnelle du 23/07/2008 pour le nouvel article 75-1 de la Constitution, déjà cité, place au contraire en haut de l'ordre juridique français le lien entre langue minoritaire historique de la France et territoire : «Les langues régionales appartiennent au patrimoine de la France $»$. Le lien au patrimoine y est renforcé, non à travers celui qui est forcément propre à chaque langue mais à travers celui, global et indifférencié, du patrimoine de la France, dans un contexte de développement de la notion de diversité culturelle en Europe et dans le monde. Cela pourrait être un paradoxe significatif d'une hésitation ou d'un rappel à l'ordre frileux. On revient au lien au territoire qui avait été critiqué auparavant, et on affirme la sujétion du régional. Il ne s'appartient pas à lui en même temps qu'à la France, il n'appartient qu'à la France. Une phrase telle que « les langues de France appartiennent au patrimoine de la France » aurait été inutilement redondante et aurait plutôt commandé un agencement et un contenu définissant un autre type d'appartenance, ou bien une déclaration tout simplement plus confiante et généreuse.

Il se dégage finalement de cet article 75-1, placé sous le titre des collectivité territoriales, et non en haut de l'édifice constitutionnel parmi les éléments constitutifs de la nation globale, française, un sentiment d'embarras qui ne pouvait déboucher que sur un aveu de stérilité trois ans plus tard confirmé par le Conseil constitutionnel ${ }^{24}$. On remarquera que l'article 3 de la Constitution espagnole de 1978 va également dans le même sens en assignant les «langues espagnoles » à des territoires (les communautés autonomes) et en les réunissant sous la bannière du patrimoine espagnol ${ }^{25}$. Néanmoins, histoires et contextes certes différents, s'il y a des ressemblances de fond entre ces formulations, les nuances qui les distinguent sont consistantes car elles ont accompagné un déclin des «langues régionales » en France et efficacement contribué à une revitalisation des « autres langues espagnoles » en Espagne ${ }^{26}$.

\section{Références bibliographiques}

Becat Joan, 2008, «Activités et autorités toponymiques en Catalogne Nord », in Hervé Guillorel (dir.), Toponymie et politique. Les marqueurs linguistiques du territoire, Bruxelles : Bruylant, pp. 41-60.

Bertile Véronique, 2010, «L'approche juridique française du rapport langue/espace », in Alain Viaut et Joël Pailhé (dirs), Langue et espace, Bordeaux: Maison des sciences de l'homme d'Aquitaine, pp. 69-84.

Carcassonne Guy, 1998, Étude sur la compatibilité entre la Charte européenne des langues régionales ou minoritaires et la Constitution, Paris: Association pour une culture

\footnotetext{
${ }^{24}$ Cf. le considérant $n^{\circ} 3$ de la Décision 2011-130 du 20/05/2011 du Conseil constitutionnel en réponse à la Question prioritaire de constitutionnalité posée par Mme Cécile L., Mme Cécile C., l'Association pour le bilinguisme franco-allemand en Moselle, l'association Culture et bilinguisme de Lorraine - Zweisprachig, unsere Zukunft et l'association Comité fédéral des associations pour la langue et la culture régionales d'Alsace « Fer unsri Zukunft ", relative à la conformité aux droits et libertés que la Constitution garantit des dispositions de l'article L. 312-10 du code de l'éducation: "Considérant qu'aux termes de l'article 75-1 de la Constitution : "Les langues régionales appartiennent au patrimoine de la France"; que cet article n'institue pas un droit ou une liberté que la Constitution garantit ; (...)» (https://www.conseil-constitutionnel.fr/decision/2011/2011130QPC.htm, consulté le 29/02/2020).

${ }^{25}$ « La richesse des diverses modalités linguistiques de l'Espagne est un patrimoine culturel qui fera l'objet d'un respect particulier et de protection » (article 3.3).

${ }^{26}$ Le castillan étant la langue espagnole officielle de l’État (article 3.1).
} 
différente, https://www.vie-publique.fr/sites/default/files/rapport/pdf/984001697.pdf (consulté le 29/02/2020).

Cerquiglini Bernard, 1999, Les langues de la France : rapport au Ministre de l'Éducation, de la Recherche et de la Technologie et à la Ministre de la Culture et de la Communication, avril 1999, https://www.vie-publique.fr/sites/default/files/rapport/pdf/994000719.pdf (consulté le 29/02/2020).

Code de l'Éducation, 2020 version consolidée [2000], Paris: Ministère de l'Éducation nationale, https://www.legifrance.gouv.fr/affichCode.do?cidTexte=LEGITEXT000006071191 (consulté le 29/02/2020).

Eysseric Violaine, 2005, Le corpus juridique des langues de France, Paris: Délégation générale à la langue française et aux langues de France, https://www.culturebilinguisme-lorraine.org/images/pdf/legislationldf.pdf (consulté le 29/02/2020).

Lejeune Sylvie, 2008, " La politique toponymique de la France et les langues régionales », in Hervé Guillorel (dir.), Toponymie et politique. Les marqueurs linguistiques $d u$ territoire, Bruxelles : Bruylant, pp. 11-33.

Poignant Bernard, 1998, Langues et cultures régionales. Rapport de monsieur Bernard Poignant Maire de Quimper à Monsieur Lionel Jospin Premier Ministre, Paris, https://www.vie-publique.fr/sites/default/files/rapport/pdf/984001448.pdf (consulté le 29/02/2020).

Sibille Jean, 2013, «La notion de langues de France, son contenu et ses limites », in Georg Kremnitz, avec le concours de Fañch Broudic et du coll. HSLF (dir.), Histoire sociale des langues de France, Rennes : Presses universitaires de Rennes, pp. 45-60.

Sibille Jean, 2010, "Langues de France" et territoires: raison des choix et des dénominations », in Alain Viaut et Joël Pailhé, Langue et espace, Bordeaux : Maison des sciences de l'homme d'Aquitaine, pp. 85-107.

Swann Joan, Deumert Ana, Lillis Theresa, Mesthrie Rajend, 2004, A Dictionary of Sociolinguistics, Edinburgh : Edinburgh University Press.

Viaut, Alain \& Pascaud, Antoine, 2017, «Pour une définition de la notion de "langue régionale" ", Lengas revue de sociolinguistique, $\mathrm{n}^{\circ} 82$, https://journals.openedition.org/lengas/1380 (consulté le 29/02/2020). 


\section{GLOTTOPOL}

Revue de sociolinguistique en ligne

Comité de rédaction : Michaël Abecassis, Salih Akin, Sophie Babault, Claude Caitucoli, Véronique Castellotti, Régine Delamotte, Robert Fournier, Stéphanie Galligani, Emmanuelle Huver, Normand Labrie, Foued Laroussi, Benoit Leblanc, Fabienne Leconte, Gudrun Ledegen, Danièle Moore, Clara Mortamet, Alioune Ndao, Isabelle Pierozak, Gisèle Prignitz.

Rédactrice en chef : Clara Mortamet.

Comité scientifique : Claudine Bavoux, Michel Beniamino, Jacqueline Billiez, Philippe Blanchet, Pierre Bouchard, Ahmed Boukous, Pierre Dumont, Jean-Michel Eloy, Françoise Gadet, Monica Heller, Caroline Juilliard, Jean-Marie Klinkenberg, Jean Le Dû $(\dagger)$, Marinette Matthey, Jacques Maurais, Marie-Louise Moreau, Robert Nicolaï, Didier de Robillard, Paul Siblot, Claude Truchot, Daniel Véronique.

\section{Comité de lecture pour ce numéro :}

Salih Akin, Carmen Alén Garabato, Sophie Babault, Philippe Blanchet, Henri Boyer, Véronique Castellotti, Marisa Cavalli, Jean-François De Pietro, Didier de Robillard, Alain Di Meglio, Ksenija Djordjevic, Jean Michel Eloy, Pascale Erahrt, Véronique Fillol, Monica Heller, Robert Fournier, Normand Labrie, Hervé Lieutard, Jean Le Dû (†), Marinette Matthey.

http://glottopol.univ-rouen.fr

ISSN : 1769-7425 